\title{
Use of anti-depressants and the risk of fracture of the hip or femur
}

\author{
M. W. M. van den Brand • M. M. Samson • S. Pouwels • \\ T. P. van Staa • B. Thio • C. Cooper • H. G. M. Leufkens • \\ A. C. G. Egberts • H. J. J. Verhaar • F. de Vries
}

Received: 27 September 2008 / Accepted: 16 January 2009 / Published online: 24 February 2009

(C) The Author(s) 2009. This article is published with open access at Springerlink.com

\begin{abstract}
Summary Anti-depressants are used largely, but have serious side effects. We show that both selective serotonin re-uptake inhibitors (SSRIs) and tricyclic anti-depressants (TCAs) increase the risk of hip/femur fracture and that this risk is time related and depends on the degree of serotonin
\end{abstract}

M. W. M. van den Brand $(\bowtie) \cdot$ M. M. Samson $\cdot$ H. J. J. Verhaar Department of Geriatric Medicine,

University Medical Centre Utrecht,

Heidelberglaan 100 ,

3584 CX Utrecht, The Netherlands

e-mail: mwm.vandenbrand@wanadoo.nl

S. Pouwels • T. P. van Staa • B. Thio • H. G. M. Leufkens •

A. C. G. Egberts $\cdot$ F. de Vries $(\bowtie)$

Division of Pharmacoepidemiology and Pharmacotherapy,

Utrecht Institute for Pharmaceutical Sciences, Universiteit Utrecht,

Sorbonnelaan 16 ,

3584 CA Utrecht, The Netherlands

e-mail: f.devries@uu.nl

T. P. van Staa $\cdot$ C. Cooper $\cdot$ F. de Vries

Medical Research Council Epidemiology Resource Centre,

University of Southampton, Southampton General Hospital,

Southampton, UK

T. P. van Staa $\cdot$ F. de Vries

General Practice Research Database,

Medicines and Healthcare products Regulatory Agency,

19 Elmslane, SW8 5NQ,

London, UK

C. Cooper

Institute of Musculoskeletal Sciences, University of Oxford,

Oxford, UK

\section{A. C. G. Egberts}

Department of Clinical Pharmacy,

University Medical Centre Utrecht,

Heidelberglaan 100, 3584 CX,

Utrecht, The Netherlands transporter inhibition. This should be considered when prescribing anti-depressants to patients.

Introduction Anti-depressants are known to have serious side effects. We examined the association between the use of antidepressants and the risk of hip/femur fractures with a special focus on the relation with the degree of 5-hydroxytryptamine transporter (5-HTT) inhibition and the duration of use.

Methods A case-control study was conducted within the Dutch PHARMO-RLS database. Cases $(n=6,763)$ were adult patients with a first hip/femur fracture during the study period. For each case, four controls $(n=26341)$ were matched by age, gender and geographic region.

Results The risk of hip/femur fracture increased with current use of SSRIs (adjusted odds ratio $\left(\mathrm{OR}_{\mathrm{adj}}\right) 2.35$ [95\% confidence interval (CI) 1.94-2.84]) and TCAs ( $\mathrm{OR}_{\text {adj }} 1.76$ [95\% CI 1.45-2.15]). The risk of hip/femur fracture declined rapidly after discontinuation of use. The risk of hip/femur fracture increased as the degree of 5-HTT inhibition of all anti-depressants increased from $\mathrm{OR}_{\text {adj }} 1.64$ [95\% CI 1.142.35] for drugs with low 5-HTT inhibition to $\mathrm{OR}_{\text {adj }} 2.31[95 \%$ CI 1.94-2.76] for those with high 5-HTT inhibiting properties. Conclusion Current use of both SSRIs and TCAs increase hip/femur fracture risk. Further studies are needed to elucidate the mechanistic pathways and the relation with the underlying pathophysiology. Until then, the elevated fracture risk should be considered when prescribing anti-depressants.

Keywords Duration of use · Fracture · Risk · SSRI · 5-HTT inhibition

\section{Introduction}

Depression is one of the most important mental health problems especially in the elderly and is associated with a 
poor natural history, reduced quality of life, increased utilisation of medical health services and high mortality [14]. Although depression can be treated effectively with tricyclic anti-depressants (TCAs), many users experience cardiovascular (e.g. orthostatic hypotension) and anticholinergic side effects (e.g. visual disturbances), which both may increase the risk of falling and thereby of fractures. The newer generation of anti-depressants, including the selective serotonin re-uptake inhibitors (SSRIs), are considered as effective as the TCAs but with less bothersome side effects. Its use has increased over the last decade [5-7]. Some studies investigating the risk of falls with anti-depressants have reported no significant difference in risk for SSRIs and TCAs $[8,9]$.

Falls increase the risk of fracture, especially of the hip or femur in the elderly [10], with significant consequences for the individual and healthcare providers in terms of the impact on long-term morbidity, mortality and healthcare costs [11-14]. Several epidemiological studies have reported an increased risk of fracture with anti-depressant use $[9,15-17]$. One explanation is that the increased fracture risk is mediated simply by falling [8]. Another explanation lies in the potential for anti-depressants to affect the micro-architecture of bone. Functional serotonin (5-hydroxytryptamine, 5-HT) receptors and transporter systems have been localised on osteoblasts, osteoclasts and osteocytes [18-22] and 5-HT stimulates proliferation of osteoblast precursor cells in vitro [23]. Thus, drugs that block 5-HT re-uptake could affect bone metabolism and have a negative impact on bone micro-architecture. This has been illustrated by a recent case-control study conducted in Denmark, which reported an increased risk of fractures with an increased degree of blocking of the serotonin system [24].

The aim of this study was to examine the association between the use of anti-depressants and the risk of hip/ femur fractures, with a special focus on the relation with the degree of 5-hydroxytryptamine transporter (5-HTT) inhibition afforded by different anti-depressants and the duration of use.

\section{Materials and methods}

\section{Study design}

We conducted a case-control study within the Dutch PHARMO Record Linkage System (RLS) (www.pharmo.nl). The database includes the demographic details and complete medication histories for about one million community-dwelling residents in The Netherlands representing some $7 \%$ of the general population. Data are linked to hospital discharge records as well as several other health registries, including pathology, clinical laboratory findings and general practitioner data [25]. Almost every individual in The Netherlands is registered with a single community pharmacy, independent of prescriber and irrespective of their health insurance or socio-economic status. Pharmacy records have a high degree of completeness with regards to dispensed drugs [26, 27]. Pharmacy data include information about the drug dispensed, the date of dispensing, the prescriber, the amount dispensed, the prescribed dosage regimen and the estimated duration of use. Hospital discharge records include detailed information on date of admission, discharge diagnoses and procedures. Validation studies on PHARMO RLS have confirmed a high level of data completeness and validity [28-30]. During data collection, the privacy and confidentiality of patients is maintained and complies with the Dutch Data Protection Act.

\section{Study population}

Data were collected for the period 1 January 1991 to 31 December 2002. Cases were patients aged 18 years and older with a record for a first fracture of the hip or femur during the study period. The date of hospital admission was used to define the index date. Each case was matched by year of birth, sex and geographical region to up to four control patients without any evidence of ever having sustained a fracture. The index date for each control was the same as the date of fracture for the matched case.

\section{Exposure assessment}

Exposure to anti-depressants was determined by reviewing prescription information before the index date. Current users were defined as individuals who had received a prescription for a TCA, an SSRI or other anti-depressant within a 30-day period before the index date. Recent users were individuals whose most recent prescription was issued 31-90 days before the index date, and past users were those whose most recent prescription had been issued more than 3 months ( $>90$ days) before the index date. Patients with a history of using more than one type of anti-depressant before the index date were classified as appropriate, e.g. a current user of an SSRI may also qualify as a current user of a TCA. The average daily dose was calculated by dividing the cumulative exposure by the total treatment time. Dose equivalencies of anti-depressants were applied from the WHO defined daily dose (DDD) [31] and were expressed as paroxetine equivalents (SSRIs) or amitriptyline equivalents (TCAs). The extent of 5-HTT inhibition was determined for each anti-depressant with reference to Goodman and Gilman's 'The Pharmacological Basis of Therapeutics' [32] (Table 1). 
Table 1 Drugs grouped according to the degree of serotonin transporter inhibition [31]

\begin{tabular}{llll}
\hline Degree of serotonin transporter inhibition (inhibition constant in $\mathrm{nM})$ & \\
\hline Low $(>10)$ & Intermediate $(>1 \leq 10)$ & High $(\leq 1)$ & Not classified \\
\hline Desipramine & Imipramine & Clomipramine & Opipramol \\
Nortriptyline & Amitriptyline & Fluoxetine & Dosulepin \\
Doxepine & Fluvoxamine & Paroxetine & Moclobemide \\
Maprotiline & Venlafaxine & Sertraline & \\
Mianserine & Citalopram & & \\
Trazodone & & & \\
Nefadozone & & & \\
Mirtazapine & & & \\
\hline
\end{tabular}

For each prescription, the expected duration of use (in days) was based on how the drug was supplied and the prescribed daily dose. If there were missing data on the total drug supply or written dosage instruction, the expected duration of use (based on the median duration for a prescription from patients of similar age and sex) was taken. When repeat prescriptions were issued, the expected duration of use period was extended according to the expected duration of the repeat prescription. In the event of overlap between two prescriptions (i.e. a repeat prescription given before the expected end date of a previous prescription), the 'overlap' days were added to the theoretical end date of the repeat prescription. If the gap between any consecutive prescriptions was 6 months or less, exposure was deemed to be continuous.

\section{Potential confounders}

The records of cases and controls were assessed for potential confounding variables, including use within the 3 months before the index date of a benzodiazepine; use within the 6 months before the index date of an anti-psychotic (other than lithium), lithium, an anti-Parkinson drug, anticonvulsant, oral or inhaled glucocorticoid, bronchodilator, hormone replacement therapy, a disease-modifying antirheumatic drug (DMARD), anti-arrhythmic, thiazide diuretic, beta-blocker, drug for diabetes, metoclopramide, morphine/ opiate or two or more prescriptions for an non-steroidal antiinflammatory drug (NSAID); and a history at any time of hospitalisation for cardiovascular disease, malignant neoplasm, inflammatory bowel disease, rheumatoid disease, obstructive airway disease, impaired renal function, mental disorder or cerebrovascular disease. We have chosen a different time window for benzodiazepines, because in The Netherlands, benzodiazepines are dispensed for periods up to 1 month and other drugs for periods up to 3 months.

Statistical analysis

Conditional logistic regression analysis was used to estimate the risk of hip/femur fracture associated with the use of TCAs, SSRIs and the various confounding variables (SAS version 9.1.3, PHREG procedure) and were expressed as odds ratios (OR) with corresponding 95\% confidence intervals $(\mathrm{CI})$. Adjusted odds ratios $\left(\mathrm{OR}_{\mathrm{adj}}\right)$ for hip/femur fracture were estimated by comparing antidepressant use with no use using conditional logistic regression analysis. Final regression models were determined by stepwise backward elimination using a significance level of 0.05 . We stratified the study population to assess the risk with current use by age and sex.

Further analyses were conducted to evaluate the risk of fracture associated with current exposure to antidepressants versus no use grouping current users according to the daily dose of anti-depressant prescribed and according to the degree of 5-HTT inhibition expected. Smoothing spline regression plots (SAS version 9.1.3) were used to visualise the longitudinal relationship between the risk of fracture and (a) the time between the index date and last dispensing of an anti-depressant (recency of use) and (b) the duration of continuous use. The population attributable risk (PAR) was estimated using the following formula:

$$
\operatorname{PAR} \%=\frac{\operatorname{Pe}(\mathrm{OR}-1)}{1+\operatorname{Pe}(\mathrm{OR}-1)} \times 100 \text {. }
$$

The prevalence $(\mathrm{Pe})$ of anti-depressant use was derived from national prescribing figures in 2003, www.gipdatabank.nl.

\section{Results}

We identified 6,763 patients who suffered a hip/femur fracture. These cases were matched to 26,341 controls. The mean age of cases and controls was 75 years and $73 \%$ were female (Table 2). The mean period of time with prescription information before the index date was 4.1 years. Prescriptions for paroxetine accounted for $50 \%$ of the prescriptions issued for an SSRI $(25,131 / 50,287)$. Most of the other SSRI prescriptions were for fluoxetine $(23.4 \%)$ or fluvoxamine (20.3\%). Amitriptyline (46.6\%) and clomipramine (23.1\%) 
Table 2 Baseline characteristics of the study population

TCAs tricyclic anti-depressants, SSRIs selective serotonin re-uptake inhibitors, $G C s$ glucocorticosteroids

${ }^{\text {a }}$ Within the 6 months before the index date

${ }^{\mathrm{b}}$ Within the 3 months before the index date

\begin{tabular}{|c|c|c|c|}
\hline & Cases $(n=6,763)$ & Controls $(n=26,341)$ & Crude odds ratio $(95 \% \mathrm{CI})$ \\
\hline \multicolumn{4}{|l|}{ Age } \\
\hline 18 to 49 years & $452(6.7 \%)$ & $1,808(6.9 \%)$ & \\
\hline 50 to 69 years & $1,061(15.7 \%)$ & $4,239(16.1 \%)$ & \\
\hline$\geq 70$ years & $5,250(77.6 \%)$ & $20,294(77.0 \%)$ & \\
\hline Mean age in years & 75.7 & 75.3 & \\
\hline Number of females & $4,929(72.9 \%)$ & $19,138(72.7 \%)$ & \\
\hline \multicolumn{4}{|c|}{ Drug use before the index date } \\
\hline TCAs & $256(3.8 \%)$ & $591(2.2 \%)$ & $1.75(1.51-2.04)$ \\
\hline SSRIs & $315(4.7 \%)$ & $582(2.2 \%)$ & $2.20(1.91-2.54)$ \\
\hline Anti-psychotics ${ }^{\mathrm{a}}$ & $412(6.1 \%)$ & $921(3.5 \%)$ & $1.79(1.58-2.02)$ \\
\hline Anti-convulsants $^{\mathrm{a}}$ & $242(3.6 \%)$ & $431(1.6 \%)$ & $2.23(1.90-2.61)$ \\
\hline Benzodiazepines $^{\mathrm{b}}$ & $967(14.3 \%)$ & $2,751(10.4 \%)$ & $1.44(1.33-1.56)$ \\
\hline Oral glucocorticosteroids ${ }^{\mathrm{a}}$ & $366(5.4 \%)$ & $918(3.5 \%)$ & $1.59(1.40-1.80)$ \\
\hline Thiazide diuretics $^{\mathrm{a}}$ & $146(2.2 \%)$ & $557(2.1 \%)$ & $1.01(0.84-1.21)$ \\
\hline Opiates $^{\mathrm{a}}$ & $253(3.7 \%)$ & $455(1.7 \%)$ & $2.24(1.92-2.63)$ \\
\hline Anti-Parkinson drugs ${ }^{\mathrm{a}}$ & $397(5.9 \%)$ & $833(3.2 \%)$ & $1.94(1.71-2.19)$ \\
\hline$\geq 2$ NSAID prescriptions ${ }^{\mathrm{a}}$ & $929(13.7 \%)$ & $2,584(9.8 \%)$ & $1.46(1.35-1.59)$ \\
\hline \multicolumn{4}{|c|}{ Hospitalisation before the index date } \\
\hline Cardiovascular disease & $359(5.3 \%)$ & $1,289(4.9 \%)$ & $1.10(0.98-1.25)$ \\
\hline Cerebrovascular disease & $296(4.4 \%)$ & $565(2.1 \%)$ & $2.12(1.84-2.45)$ \\
\hline Malignant neoplasms & $341(5.0 \%)$ & $1,021(3.9 \%)$ & $1.54(1.37-1.74)$ \\
\hline
\end{tabular}

accounted for the majority of TCA prescriptions $(n=$ 59,836).

Table 2 shows that compared with controls, cases were significantly more likely to have used a benzodiazepine in the previous 3 months and/or an anti-depressant, an antipsychotic, anti-convulsant, oral glucocorticoid, opiate or drug for Parkinson's disease within the previous 6 months. In addition, cases were significantly more likely than controls to have a history of cerebrovascular disease or malignant neoplasm.

Table 3 provides crude and adjusted risk estimates for hip/femur fracture associated with anti-depressant use according to recency of use, and the results of analyses amongst current users stratified by sex and age. Compared with individuals who had never used the anti-depressant in question, the risk of hip/femur fracture increased with current use of SSRIs (crude OR 2.88 [95\% CI 2.40-3.46]) and TCAs (crude OR 2.22 [95\% CI 1.84-2.68]). After adjustment for other variables associated with fracture risk, the ORs remained significantly increased $\left(\mathrm{OR}_{\mathrm{adj}} 2.35\right.$ [95\% CI 1.94-2.84] for SSRIs and 1.76 [95\% CI 1.45-2.15] for TCAs). Under the assumption that the risk of hip fracture amongst users of SSRIs/TCAs is similar in the period 1991-2002 and 2003, we estimated that the population attributable risk of hip fracture is $1.1 \%$ for current users of TCAs and $4.4 \%$ for current users of SSRIs. For SSRIs, there was some effect modification by sex $\left(\mathrm{OR}_{\mathrm{adj}} 2.50\right.$ [95\% CI 2.03-3.08] for females and 1.72 [95\% CI 1.08-
2.74] for males) and age $\left(\mathrm{OR}_{\mathrm{adj}} 2.00\right.$ [95\% CI 1.21-3.29] for SSRI users aged 18-69 years and 2.39 [95\% CI 1.942.94] for SSRI users aged $\geq 70$ years).

Figure 1a shows a clear association between the time since the last dispensing of an SSRI and the risk of hip/ femur fracture. The risk of hip/femur fracture, which was increased in current users, declined rapidly after discontinuation of use. A similar trend was observed for users of TCAs (Fig. 1b). The risk of hip/femur fracture was increased during the first few months of continuous use of SSRIs, peaking at about 8 months, and remained elevated after about 1.5 years of continuous use (Fig. 2a). Short-term exposure to TCAs showed a rapid increase in hip/femur fracture risk that declined after 1 year of exposure (Fig. 2b).

Table 4 presents the results of analysis amongst current users according to the average daily dose of anti-depressant used. Compared with individuals who had never used an SSRI, medium and high dose SSRI users had a greater risk of fracture than low dose users, although the differences were not statistically significant. There was no evidence to suggest a dose-response relationship for the risk of hip/ femur fracture with TCA use.

Table 5 presents the results of analyses amongst all antidepressant users, where current users were grouped according to the degree of 5-HTT inhibition afforded by the different drugs. The risk of hip/femur fracture increased as the degree of 5-HTT inhibition increased from $\mathrm{OR}_{\mathrm{adj}}$ 1.64 [95\% CI 1.14-2.35] for drugs with low 5-HTT 
Table 3 Use of SSRIs and TCAs and the risk of hip/femur fracture

\begin{tabular}{|c|c|c|c|c|c|c|}
\hline & $\begin{array}{l}\text { Cases } \\
(n=6,763)\end{array}$ & $\begin{array}{l}\text { Controls } \\
(n=26,341)\end{array}$ & $\begin{array}{l}\text { Crude } \\
\text { OR }\end{array}$ & $95 \% \mathrm{CI}$ & $\begin{array}{l}\text { Adjusted } \\
\mathrm{OR}^{\mathrm{a}}\end{array}$ & $95 \% \mathrm{CI}$ \\
\hline \multicolumn{7}{|l|}{ Exposure to SSRIs } \\
\hline Never exposed & 6,174 & 25,041 & 1.00 & - & Referent & \\
\hline Past use ( $>90$ days before the index date) & 303 & 820 & 1.52 & $1.33-1.75$ & 1.23 & $1.07-1.42$ \\
\hline Recent use (31-90 days before the index date) & 86 & 193 & 1.87 & $1.45-2.42$ & 1.48 & $1.14-1.93$ \\
\hline Current use (1-30 days before the index date) & 200 & 287 & 2.88 & $2.40-3.46$ & 2.35 & $1.94-2.84$ \\
\hline Males & 33 & 59 & 2.30 & $1.48-3.59$ & 1.72 & $1.08-2.74$ \\
\hline Females & 167 & 228 & 3.02 & $2.46-3.70$ & 2.50 & $2.03-3.08$ \\
\hline Age $18-69$ years & 33 & 44 & 3.07 & $1.95-4.82$ & 2.00 & $1.21-3.29$ \\
\hline Age $\geq 70$ years & 167 & 243 & 2.84 & $2.32-3.48$ & 2.39 & $1.94-2.94$ \\
\hline \multicolumn{7}{|l|}{ Exposure to TCAs } \\
\hline Never exposed & 6,175 & 24,864 & 1.00 & - & Referent & \\
\hline Past use ( $>90$ days before the index date) & 360 & 978 & 1.52 & $1.34-1.72$ & 1.14 & $1.00-1.30$ \\
\hline Recent use (31-90 days before the index date) & 56 & 176 & 1.32 & $0.97-1.79$ & 0.98 & $0.72-1.34$ \\
\hline Current use (1-30 days before the index date) & 172 & 323 & 2.22 & $1.84-2.68$ & 1.76 & $1.45-2.15$ \\
\hline Males & 29 & 43 & 2.85 & $1.76-4.62$ & 2.19 & $1.31-3.67$ \\
\hline Females & 143 & 280 & 2.11 & $1.72-2.60$ & 1.69 & $1.36-2.09$ \\
\hline Age $18-69$ years & 31 & 60 & 2.18 & $1.40-3.40$ & 1.31 & $0.80-2.14$ \\
\hline Age $\geq 70$ years & 141 & 263 & 2.22 & $1.80-2.74$ & 1.81 & $1.46-2.25$ \\
\hline
\end{tabular}

${ }^{a}$ SSRI use was adjusted for (a) current use of an anti-depressant other than SSRI, (b) use in the past 3 months of a benzodiazepine; (c) use in the past 6 months of oral corticosteroids, hormone replacement therapy, anti-psychotics, beta-blockers, opioids, anti-convulsants, drugs for diabetes, more than two dispensings of an NSAID, DMARDs and metoclopramide and (d) a history of malignant neoplasms, mental disorders, cerebrovascular diseases, obstructive airway diseases or inflammatory bowel diseases. TCA use was adjusted for current use of an anti-depressant other than TCA and other potential confounders as listed above (b)-(d) for SSRI use

inhibition to $\mathrm{OR}_{\text {adj }} 2.31$ [95\% CI 1.94-2.76] for those with high 5-HTT inhibiting properties. Users of anti-depressants with stronger anti-cholinergic properties, or a strong potential to induce orthostatic hypotension, did not have higher risks of hip fracture compared to users of antidepressants with weaker properties (data not shown).

\section{Discussion}

This study has demonstrated an increased risk hip/femur fracture for current users of SSRIs and TCAs. For both SSRIs and TCAs, the increased risk declined rapidly about 6 months after discontinuation of use. Fracture risk associated with SSRIs and TCAs was the greatest during the first few months of use and an elevated risk persisted with continuous use of SSRIs. We found some evidence for a dose effect with SSRIs but not TCAs. Furthermore, we found evidence to suggest that the risk of fracture was greater amongst people using anti-depressants with a higher degree of 5-HTT inhibition.

The magnitude of increased fracture risk with antidepressant use described here is in line with findings from other epidemiological studies [9, 15-17, 24]. Those studies that compared risk with SSRIs and TCAs $[9,15,16]$ similarly reported no difference in risk. There is also evidence to support our observation of an increased risk during the initial period of exposure $[15,16]$. Richards et al. [17] investigated fracture risk with SSRIs and reported a dose effect and a sustained elevation in risk with prolonged use. Vestergaard et al. reported a dose-dependent increase in fracture risk for sedating TCAs and most SSRIs. Furthermore, they also found an association between the increase in risk of any fracture and the inhibition of the serotonin transporter system [24].

We observed a similar increase in fracture risk for users of SSRIs and TCAs. The explanation for that increased fracture risk may be related simply to an increase in the risk of falls associated with anti-depressant use, especially as there is evidence to suggest that both SSRIs and TCAs are associated with an increased risk of fall. A large study of nursing home residents showed that, compared with nonusers and after adjusting for potential confounders, the risk of falls was similar in new users of TCAs and SSRIs. The association was dose dependent and the increased risk persisted through the first 180 days of use and beyond [8]. TCAs are known to inhibit cardiovascular $\mathrm{Na}+, \mathrm{Ca} 2+$ and $\mathrm{K}+$ channels which can lead to life-threatening arrhythmias. SSRI use has been associated with an increased risk of syncope [33], postural hypotension and dizziness [34] 

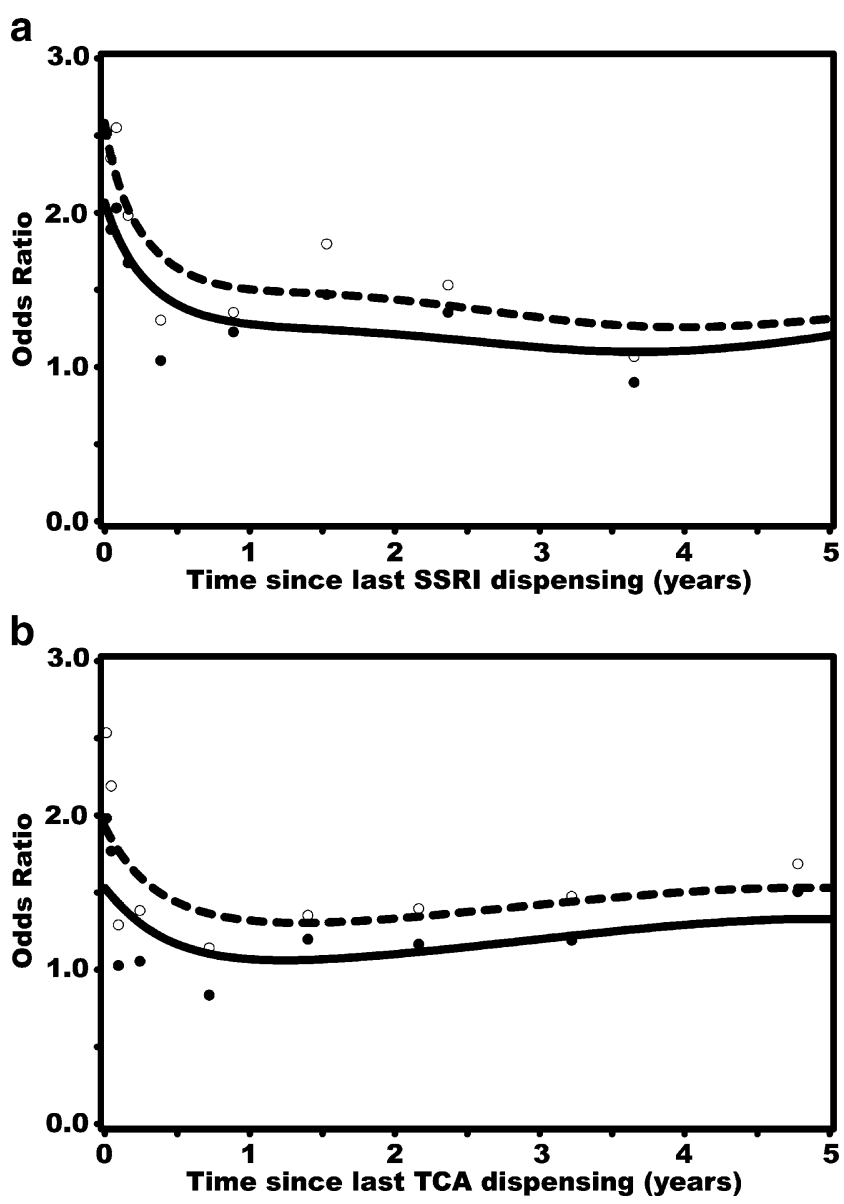

Fig. 1 a, b Recency of SSRI (a) and TCA (b) use before the index date and risk of hip/femur fracture. Dashed lines and open dots: crude ORs with 95\% CI; solid lines and solid dots: adjusted ORs with $95 \%$ CI. Adjustments were made for the same confounders as in Table 3

during the early days of exposure, and both SSRIs and TCAs can affect sleep patterns $[35,36]$, thereby increasing the risk of falls [37].

Another explanation for the increased fracture risk observed here is the effect of anti-depressants on bone physiology. Functional 5-HT receptors are present in bone cells and 5-HT stimulates proliferation of osteoblast precursor cells in vitro [23]. There is emerging evidence from animal studies that 5 -HT is involved in bone remodelling and can alter bone mineral density (BMD) [18-20, 22]. Indeed, recent findings have shown that SSRIs decrease BMD in animal models [38] and humans [17, 3941]. Such studies that compared BMD changes with different anti-depressants reported no association between TCA use and BMD [39, 40]. In a recent study of osteoporotic fractures, it was observed that the use of SSRIs (but not TCAs) in older women was independently associated with an increased rate of hip bone loss $(0.82 \%$ reduction per year) [41], although there was limited information on dose and duration of use.
To explore the possibility that fracture risk may be directly related to inhibition of the 5-HTT system, we grouped together the anti-depressants used according to the degree of 5-HTT inhibition afforded. It was apparent that the risk of hip/femur fracture increased as the degree of 5-HTT inhibition increased. Whilst none of the risk estimates was significantly different, a clear trend was evident and this supports the possibility that stronger inhibition of the 5-HTT system on the bone could cause a greater disruption of the balance between osteoblasts and osteoclasts and hence have a greater detrimental effect on bone micro-architecture.

Drug-induced changes in bone micro-architecture can be rapid. Analysis of the micro-architecture of femur bone in rats treated with 5-HT showed changes in trabecular bone volume and an increased femoral stiffness after just 3 months [10]. Other drug exposures had demonstrated similarly rapid effects on human bone, e.g. corticosteroids $[42,43]$. It is possible that a rapid change in bone microarchitecture affected by anti-depressant use accounted for,
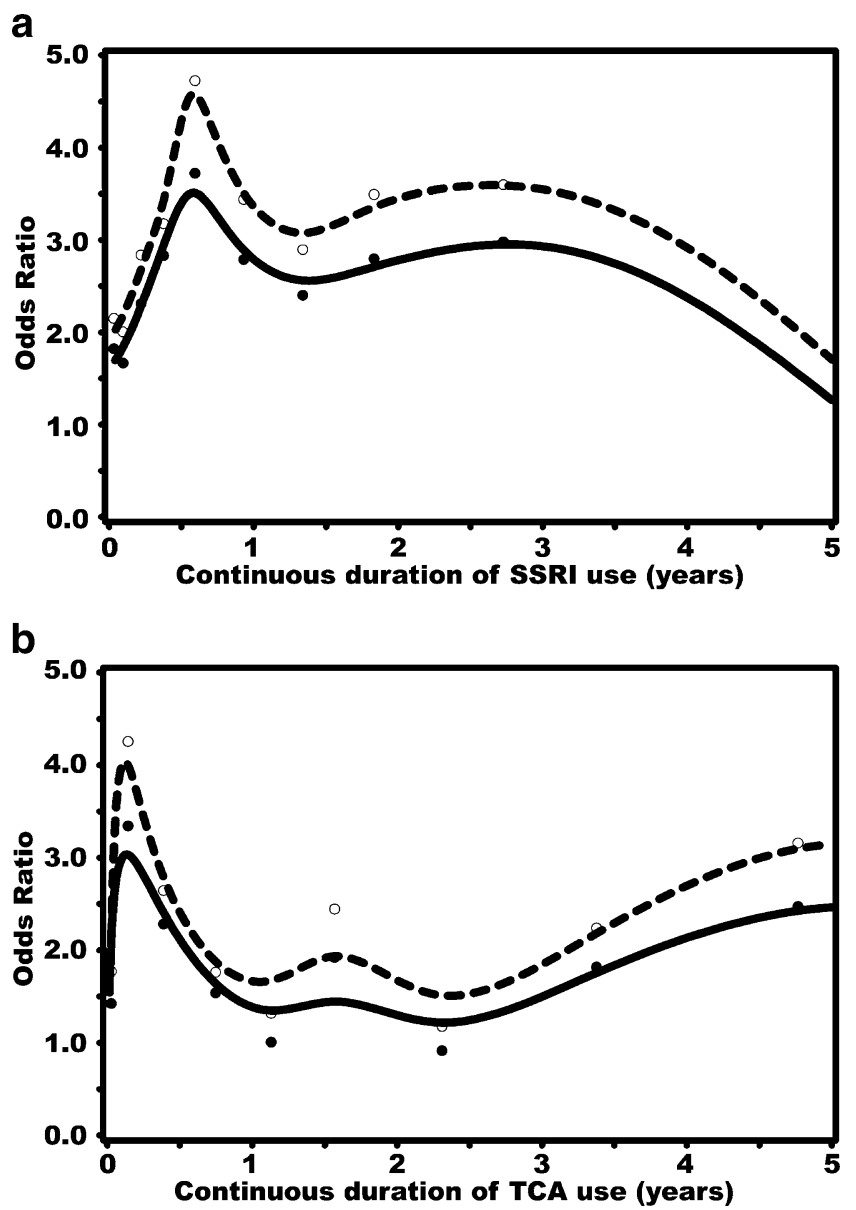

Fig. 2 a, b Recency of SSRI (a) and TCA (b) duration of continuous use amongst current users before the index date and risk of hip/femur fracture. Dashed lines and open dots: crude ORs with 95\% CI; solid lines and solid dots: adjusted ORs with 95\% CI. Adjustments were made for the same confounders as in Table 3 
Table 4 Current use of SSRIs and TCAs and the risk of hip/femur fracture by average daily dose

\begin{tabular}{|c|c|c|c|c|c|c|}
\hline Average daily dose (DDD) & Cases & Controls & Crude OR & $95 \% \mathrm{CI}$ & Adjusted $\mathrm{OR}^{\mathrm{c}}$ & $95 \% \mathrm{CI}$ \\
\hline \multicolumn{7}{|l|}{ Current SSRI use $^{\mathrm{a}}$} \\
\hline One prescription before the index date & 16 & 30 & 2.15 & $1.17-3.96$ & 1.72 & $0.92-3.21$ \\
\hline Low $(<0.5)$ & 22 & 47 & 1.88 & $1.13-3.13$ & 1.50 & $0.89-2.53$ \\
\hline Medium (0.5-1.0) & 77 & 95 & 3.40 & $2.51-4.62$ & 2.77 & $2.03-3.80$ \\
\hline High $(>1.0)$ & 85 & 115 & 3.08 & $2.31-4.09$ & 2.49 & $1.86-3.34$ \\
\hline \multicolumn{7}{|l|}{ Current TCA use $\mathrm{e}^{\mathrm{b}}$} \\
\hline One prescription before the index date & 12 & 21 & 2.39 & $1.17-4.86$ & 1.95 & $0.94-4.06$ \\
\hline Low $(<0.5)$ & 95 & 186 & 2.13 & $1.66-2.74$ & 1.73 & $1.33-2.24$ \\
\hline Medium (0.5-1.0) & 53 & 91 & 2.41 & $1.71-3.38$ & 1.82 & $1.28-2.58$ \\
\hline High $(>1.0)$ & 12 & 25 & 1.99 & $1.00-3.97$ & 1.35 & $0.66-2.79$ \\
\hline
\end{tabular}

${ }^{\mathrm{a}}$ Referent: never exposed to SSRIs

${ }^{\mathrm{b}}$ Referent: never exposed to TCAs

${ }^{\mathrm{c}}$ Adjustments were made for the confounders listed in the footnote of Table 3

or at least contributed to, the increased fracture risk during the early months of exposure.

We found that as the duration of treatment with TCAs increased, the risk of fracture declined, whereas the risk for fracture with continuation of SSRIs fell after the initial increase but remained somewhat elevated thereafter. It may be that with chronic administration of anti-depressants, adaptive changes occur [44]. These may result in an adjustment to the cardiovascular effect of TCAs and SSRIs, explaining the decrease in fracture risk after a few months of use, whereas changes in bone physiology are not subject to adaptive changes, explaining the sustained fracture risk in SSRI users.

Limitations of our study include absence of potentially confounding data on body mass index (BMI), smoking status and exercise. In a US/Puerto Rican cohort study, it was likely that lack of adjustment for BMI, current smoking status, activities of daily living score, cognitive impairment and Rosow-Breslau physical impairment scale accounted for up to $30 \%$ of the increased risk of hip fractures amongst users of SSRIs [45]. We do not anticipate that missing data on these variables would have an important impact on our findings; therefore, as if our ORs were decreased by $30 \%$, a positive association would remain. Another limitation lies in the potential for confounding by indication, as depression itself is associated with an increased risk of falls and fractures [46]. There is also the possibility of a channelling effect whereby, for some frail patients with depression, an SSRI was prescribed instead of a TCA because of the more favourable side-effect profile anticipated. This could have overestimated the risk associated with SSRIs observed here. These unmeasured types of confounding as well as selection bias (e.g. healthy user bias), which can change over time, may be alternative explanations for our observed associations between fracture risk and duration of anti-depressant use or discontinuation of anti-depressants. In Figs. 1 and 2, data beyond 4 years are sparse, which makes extrapolation uncertain. Lastly, the PAR calculation showed that $4.4 \%$ of hip fractures in The Netherlands might be attributed to current use of SSRI; however, given the previous limitations and the susceptibility of our study for unmeasured distortions, this figure should be interpreted with great care.

Table 5 Risk of hip/femur fracture by degree of serotonin (5-HT) transporter inhibition

\begin{tabular}{|c|c|c|c|c|}
\hline & Cases $(n=6,763)$ & Controls $(n=26,341)$ & Adjusted $\mathrm{OR}^{\mathrm{a}}$ & $95 \% \mathrm{CI}$ \\
\hline Never exposed & 5,677 & 23,698 & Referent & - \\
\hline Past use ( $>90$ days before the index date) & 506 & 1,514 & 1.19 & $1.76-2.29$ \\
\hline Recent use (31-90 days before the index date) & 158 & 404 & 1.32 & $1.09-1.61$ \\
\hline Current use ( $1-30$ days before the index date) & 422 & 725 & 2.01 & $1.76-1.29$ \\
\hline Low 5-HT transporter inhibition & 46 & 102 & 1.64 & $1.14-2.35$ \\
\hline Medium 5-HT transporter inhibition & 132 & 241 & 1.92 & $1.53-2.40$ \\
\hline High 5-HT transporter inhibition & 234 & 358 & 2.31 & $1.94-2.76$ \\
\hline Not classified & 10 & 24 & 1.44 & $0.67-3.04$ \\
\hline
\end{tabular}

${ }^{\text {a }}$ Adjustments were made for the confounders listed in the footnote of Table 3 
One of the strengths of this study is size of the population available and the reliability of information on prescribing and hospitalisations. Furthermore, the longitudinal nature of recording has two advantages. First, to our knowledge, this is the only study where duration of use analysis has allowed speculation on the effects of anti-depressants on bone. Second, this is the second study to evaluate the effect of 5HTT inhibition on fracture risk estimates.

In summary, our findings demonstrate that both SSRIs and TCAs increase the risk of hip/femur fracture in current users and that the risk increases with the degree of 5-HTT inhibition afforded by different anti-depressants. We did not find convincing evidence for a dose effect. The pathophysiology can be fall-related and/or bone-related. Further studies, including controlled prospective trials, are needed to evaluate the relative contribution of disease-related and treatmentrelated effects to the increased risk of falls and hip/femur fractures and to elucidate the pathophysiology. Until then, physicians prescribing anti-depressants should consider the elevated risk for fractures in elderly, possibly frail, people using anti-depressants and value the rule: "start low, go slow".

Acknowledgements The authors would like to thank Dr Helen Seaman for her assistance in the preparation of this manuscript for publication.

Funding The current study has not been funded.

Conflicts of interest Dr Van Staa and Dr de Vries also work for the General Practice Research Database (GPRD). GPRD is owned by the UK Department of Health and operates within the Medicines and Healthcare products Regulatory Agency (MHRA). GPRD is funded by the MHRA, Medical Research Council, various universities, contract research organisations and pharmaceutical companies.

The division of Pharmacoepidemiology \& Pharmacotherapy employing authors SP, TS and BT, HL, AE and FV has received unrestricted funding for pharmacoepidemiological research from GlaxoSmithKline, Novo Nordisk, the private-public funded Top Institute Pharma (www.tipharma.nl, includes co-funding from universities, government and industry), the Dutch Medicines Evaluation Board and the Dutch Ministry of Health.

Open Access This article is distributed under the terms of the Creative Commons Attribution Noncommercial License which permits any noncommercial use, distribution, and reproduction in any medium, provided the original author(s) and source are credited.

\section{References}

1. Cole MG, Bellavance F, Mansour A (1999) Prognosis of depression in elderly community and primary care populations: a systematic review and meta-analysis. Am J Psychiatry 156(8):1182-1189

2. Beekman AT et al (2002) The impact of depression on the wellbeing, disability and use of services in older adults: a longitudinal perspective. Acta Psychiatr Scand 105(1):20-27
3. Cuijpers P, Smit F (2002) Excess mortality in depression: a metaanalysis of community studies. J Affect Disord 72(3):227-236

4. Unutzer J et al (1997) Depressive symptoms and the cost of health services in HMO patients aged 65 years and older. A 4-year prospective study. Jama 277(20):1618-1623

5. Meijer WE et al (2004) Incidence and determinants of long-term use of antidepressants. Eur J Clin Pharmacol 60(1):57-61

6. Rosholm JU, Andersen M, Gram LF (2001) Are there differences in the use of selective serotonin reuptake inhibitors and tricyclic antidepressants? A prescription database study. Eur J Clin Pharmacol 56(12):923-929

7. Martin RM et al (1997) General practitioners' perceptions of the tolerability of antidepressant drugs: a comparison of selective serotonin reuptake inhibitors and tricyclic antidepressants. BMJ 314(7081):646-651

8. Thapa PB et al (1998) Antidepressants and the risk of falls among nursing home residents. N Engl J Med 339(13):875-882

9. Ensrud KE et al (2003) Central nervous system active medications and risk for fractures in older women. Arch Intern Med 163(8):949-957

10. Gustafsson BI et al (2006) Long-term serotonin administration leads to higher bone mineral density, affects bone architecture, and leads to higher femoral bone stiffness in rats. J Cell Biochem 97 (6):1283-1291

11. Braithwaite RS, Col NF, Wong JB (2003) Estimating hip fracture morbidity, mortality and costs. J Am Geriatr Soc 51(3):364-370

12. Haentjens P, Lamraski G, Boonen S (2005) Costs and consequences of hip fracture occurrence in old age: an economic perspective. Disabil Rehabil 27(18-19):1129-1141

13. Keene GS, Parker MJ, Pryor GA (1993) Mortality and morbidity after hip fractures. BMJ 307(6914):1248-1250

14. Roche JJ et al (2005) Effect of comorbidities and postoperative complications on mortality after hip fracture in elderly people: prospective observational cohort study. BMJ 331(7529):1374

15. Hubbard R et al (2003) Exposure to tricyclic and selective serotonin reuptake inhibitor antidepressants and the risk of hip fracture. Am J Epidemiol 158(1):77-84

16. Liu B et al (1998) Use of selective serotonin-reuptake inhibitors of tricyclic antidepressants and risk of hip fractures in elderly people. Lancet 351(9112):1303-1307

17. Richards JB et al (2007) Effect of selective serotonin reuptake inhibitors on the risk of fracture. Arch Intern Med 167(2):188 194

18. Battaglino R et al (2004) Serotonin regulates osteoclast differentiation through its transporter. J Bone Miner Res 19(9):1420-1431

19. Bliziotes $\mathrm{M}$ et al (2002) The role of dopamine and serotonin in regulating bone mass and strength: studies on dopamine and serotonin transporter null mice. J Musculoskelet Neuronal Interact 2(3):291-295

20. Bliziotes $M$ et al (2006) Serotonin transporter and receptor expression in osteocytic MLO-Y4 cells. Bone 39(6):1313-1321

21. Bliziotes MM et al (2001) Neurotransmitter action in osteoblasts: expression of a functional system for serotonin receptor activation and reuptake. Bone 29(5):477-486

22. Westbroek I et al (2001) Expression of serotonin receptors in bone. J Biol Chem 276(31):28961-28968

23. Gustafsson BI et al (2006) Serotonin and fluoxetine modulate bone cell function in vitro. J Cell Biochem 98(1):139-151

24. Vestergaard $P$ et al (2008) Selective serotonin reuptake inhibitors and other antidepressants and risk of fracture. Calcif Tissue Int 82:92-101

25. Herings R (1993) The PHARMO Drug Data Base: design and structure. PHARMO, a record linkage system for post-marketing surveillance of prescription drugs in The Netherlands. Doctoral thesis, Utrecht University, Utrecht, The Netherlands, pp 17-32

26. Buurma H, De Smet PA, Egberts AC (2006) Clinical risk management in Dutch community pharmacies: the case of drugdrug interactions. Drug Saf 29(8):723-732 
27. Van der Schee E, Groenewegen PP, Friele RD (2006) Public trust in health care: a performance indicator? J Health Organ Manag 20 (5):468-476

28. Van Staa TP et al (2000) Use of oral corticosteroids and risk of fractures. J Bone Miner Res 15(6):993-1000

29. Herings RM et al (1996) Current use of thiazide diuretics and prevention of femur fractures. J Clin Epidemiol 49(1):115-119

30. Heerdink ER et al (1998) NSAIDs associated with increased risk of congestive heart failure in elderly patients taking diuretics. Arch Intern Med 158(10):1108-1112

31. WHO Collaborating Centre for Drug Statistics Methodology, Norwegian Institute of Public Health (2002) ATC classification index with DDDs 2002. WHO Collaborating Centre for Drug Statistics Methodology, Norwegian Institute of Public Health, Nydalen

32. Baldessarini R (2001) Drugs and the treatment of psychiatric disorders. Depression and anxiety disorders. Chapter 19, in Goodman \& Gilman's. In: Hardman J, Limbird L, Goodman A (eds) The pharmacological basis of therapeutics. McGraw-Hill, New York, p 456

33. Cherin P et al (1997) Risk of syncope in the elderly and consumption of drugs: a case-control study. J Clin Epidemiol 50 (3):313-320

34. Laursen AL et al (1985) Paroxetine in the treatment of depressiona randomized comparison with amitriptyline. Acta Psychiatr Scand 71(3):249-255

35. Ensrud KE et al (2006) Use of selective serotonin reuptake inhibitors and sleep disturbances in community-dwelling older women. J Am Geriatr Soc 54(10):1508-1515

36. Newman AB et al (1997) Sleep disturbance, psychosocial correlates, and cardiovascular disease in 5201 older adults: the Cardiovascular Health Study. J Am Geriatr Soc 45(1):1-7
37. Stone KL et al (2006) Self-reported sleep and nap habits and risk of falls and fractures in older women: the study of osteoporotic fractures. J Am Geriatr Soc 54(8):1177-1183

38. Warden SJ et al (2005) Inhibition of the serotonin (5-hydroxytryptamine) transporter reduces bone accrual during growth. Endocrinology 146(2):685-693

39. Cauley JA et al (2005) Factors associated with the lumbar spine and proximal femur bone mineral density in older men. Osteoporos Int 16(12):1525-1537

40. Haney EM et al (2007) Association of low bone mineral density with selective serotonin reuptake inhibitor use by older men. Arch Intern Med 167(12):1246-1251

41. Diem SJ et al (2007) Use of antidepressants and rates of hip bone loss in older women: the study of osteoporotic fractures. Arch Intern Med 167(12):1240-1245

42. Manolagas SC (2000) Corticosteroids and fractures: a close encounter of the third cell kind. J Bone Miner Res 15(6):1001-1005

43. Weinstein RS et al (1998) Inhibition of osteoblastogenesis and promotion of apoptosis of osteoblasts and osteocytes by glucocorticoids. Potential mechanisms of their deleterious effects on bone. J Clin Invest 102(2):274-282

44. Richelson E (2003) Interactions of antidepressants with neurotransmitter transporters and receptors and their clinical relevance. J Clin Psychiatry 64(Suppl 13):5-12

45. Schneeweiss S, Wang PS (2004) Association between SSRI use and hip fractures and the effect of residual confounding bias in claims database studies. J Clin Psychopharmacol 24(6):632-638

46. Whooley MA et al (1999) Depression, falls, and risk of fracture in older women. Study of Osteoporotic Fractures Research Group. Arch Intern Med 159(5):484-490 